\title{
ENTRE POBREZA Y VIOLENCIA: ¿CÓMO AFECTAN LOS ESTRESORES DIARIOS Y LA VIOLENCIA A ADOLESCENTES EN ZONAS DE GUERRA?
}

\section{BETWEEN POVERTY AND VIOLENCE: HOW DO DAILY STRESSORS AND EXPOSURE TO VIOLENCE AFFECT ADOLESCENTS LIVING IN A WAR ZONE?}

\author{
Cindy Mels \\ Universidad Católica del Uruguay, Uruguay
}

\begin{abstract}
Resumen: Se ha establecido científicamente que la violencia sufrida en situaciones de guerra afecta negativamente al funcionamiento psicológico de las víctimas, no obstante, se supone que es el conjunto de varios factores de riesgo y factores protectores los que determinan las reacciones concretas. Este artículo se enfocará en dos factores de riesgo -la exposición a eventos violentos y los estresores diarios- y su relación con conductas internalizadas (depresión y ansiedad) en adolescentes viviendo en una zona de guerra. A través de un muestreo por conglomerados comprendiendo 13 colegios en 4 zonas seleccionadas en Ituri, una provincia en el noreste de la República Democrática del Congo, se encuestan 991 alumnos (13 a 21 años) por medio de cuestionarios de auto-informe, revelando datos sobre las variables sexo, edad, exposición a la violencia, estresores diarios, conductas internalizadas y externalizadas. Se encontró que los adolescentes varones resultaron más expuestos a la violencia que las adolescentes mujeres. La exposición a eventos violentos también se diferenció según la edad, en ambos sexos. A su vez, los adolescentes mayores, tanto varones como mujeres, reportaron más estresores diarios que sus pares de menor edad. Un efecto de interacción entre la exposición a la violencia y estresores diarios predijo comportamientos internalizados a medida que ambas variables se acumulaban. Además, las adolescentes mujeres, mostraron más conductas internalizadas bajo la acumulación de estresores diarios que los adolescentes varones. Concluimos que los estresores diarios están asociados con mayores niveles de conductas internalizadas, sobre todo en adolescentes mujeres, y que la exposición a la violencia aumenta el efecto. A pesar de la violencia sufrida, la reducción de estresores diarios en la vida de adolescentes afectados por la guerra, podría promover su resiliencia y minimizar en cierta medida los síntomas psicológicos causados por el estrés acumulado durante la guerra.
\end{abstract}

Palabras clave: depresión; ansiedad; adolescencia; resiliencia; República Democratica del Congo

\begin{abstract}
The negative effects of the violence suffered in situations of armed conflict on psychological functioning are well established. Nonetheless, it is considered that concrete psychological reactions result for the interaction of various risk and protective factors. This article focuses on two risk factors - the exposure to violent events and daily stressors - and their relationship with internalizing conducts (depression and anxiety) in adolescents living in a war zone.

Through a clustered sample comprising 13 secondary schools in 4 selected zones in Ituri, a province in the northeast of the Democratic Republic of Congo, we gathered data in 991 pupils (13 to 21 years). By means of self-informed questionnaires we collected data on the variables sex, age, exposure to violence, daily stressors, internalizing and externalizing behaviors.

We found boys and older adolescents to be most exposed to violence. Older adolescents reported more daily stressors compared to their younger peers, yet there was no difference by age. An interaction effect between exposure to violence and daily stressors predicted internalizing behaviors. Moreover, girls reported more internalizing problems than boys when daily stressors accumulated.

We conclude that daily stressors are associated with higher symptom levels of internalizing behavior problems, particularly in girls, while the exposure to violence increases the effect. Despite violence suffered, the reduction of daily stressors in the life of adolescents affected by war could promote resilience and minimize to a certain extent the psychological symptoms caused by the stress accumulated by war.
\end{abstract}

Keywords: depression; anxiety; adolescence; resilience; Democratic Republic of Congo 


\section{INTRODUCCIÓN}

Es un hecho establecido que la exposición a la violencia se asocia con impedimentos en el desarrollo y la presencia de varios síntomas clínicos, tanto en adultos como en niños/as y adolescentes de ambos sexos (Lopez-Soler et al., 2008; Murthy, 2007; Panter-Brick, 2010). Desde una mirada psicobiológica, se ven afectadas estructuras neurológicas que son responsables de la regulación de las reacciones frente al estrés, lo que se asocia con la aparición de un trastorno específico llamado Trastorno Estrés Postraumático (TEPT) (Lopez-Soler et al., 2008). Aunque es una categoría diagnóstica muy controvertida por su conceptualización ambigua y etnocéntrica, es la que se ha investigado más en varias poblaciones afectadas por eventos violentos, tales como torturas, ataques personales o terroristas, secuestros, abuso, etc (Lynch, 2003). El ser testigo o víctima de violencia también se ha vinculado con problemas de conducta de tipo internalizada -tales como depresión, ansiedad y baja autoestima- y con conductas externalizadas como conductas antisociales, agresión y consumo de drogas, a menudo acompañadas de problemas interpersonales y un bajo rendimiento escolar (Lynch, 2003; Panter-Brick, 2010; Williams, 2007).

Éste artículo se enfocará en un tipo particular de violencia sufrido por millones de niños/as y adolescentes a través del mundo: la guerra. Aunque no hay datos concluyentes sobre cuántos niños/as están afectados actualmente por la guerra, podemos construir un marco a partir del conocimiento con que hoy en día se cuenta: en este momento hay 24 conflictos armados activos, casi todos en países en desarrollo, donde el $50 \%$ de la población está constituida por menores de edad (Murthy, 2007; Project Ploughshares, 2012).

Los eventos violentos frecuentemente sufridos por niños/as y adolescentes en situaciones de guerra conciernen principalmente a: separación de los padres, ver cuerpos muertos o mutilados, ser testigo de tiroteos, de asesinatos, ser víctima o testigo de violaciones, secuestro (en el caso de niños soldados), entre otros (Barenbaum, Ruchkin \& Schwab-Stone, 2004; Derluyn, Broekaert, Schuyten \& De Temmerman, 2004; Mels, Derluyn, Broekaert \& Rosseel, 2009).

\section{LA VIOLENCIA DE LA GUERRA, SUS CONSECUENCIAS PSICOLÓGICAS Y FACTORES PROPICIADORES}

La prevalencia de TEPT encontrada en poblaciones de niños/as y adolescentes afectados por la guerra, ha tenido valores con amplia variación, del $22 \%$ y el $90 \%$, a través de varias investigaciones (Amone-P'Olak, Garnefski \& Kraaij, 2007; Barenbaum et al., 2004; Derluyn, Broekaert, Schuyten \& De Temmerman, 2004; Entholt \& Yule, 2006). Aunque menos indagada, la prevalencia reportada de depresión ha sido informada en el $4 \%$ al $47 \%$ y entre el $11 \%$ y $57 \%$ para síntomas clínicos de ansiedad (Barenbaum et al., 2004; Ehntholt \& Yule, 2006).

Está claro que varios factores -entre ellos las características propias de cada conflicto armado- influyen en el impacto que tiene la guerra en el funcionamiento psicológico de los afectados. Entre los factores más estudiados, se encuentran el tipo, la severidad y la cantidad de eventos violentos experimentados (Barenbaum et al., 2004; Macksoud \& Aber, 1996). En cuanto a ésto, la existencia de una relación positiva entre el número de experiencias violentas prolongadas en el tiempo y la cantidad y severidad de síntomas reportados, ha sido establecida a través de varios escenarios y poblaciones (Derluyn, Mels \& Broekaert, 2009; Ehntholt \& Yule, 2006; Murthy, 2007; Porter \& Haslam, 2005).

A su vez, ciertas características individuales han sido asociadas con determinadas consecuencias psicológicas, más allá de la cantidad de eventos violentos sufridos. Los datos sobre el papel del sexo han mostrado sistemáticamente, que las adolescentes mujeres tienden a presentar más sintomatología que los adolescentes varones, sobre todo en lo que concierne a los síntomas vinculados a TEPT y conductas internalizadas (Barenbaum et al., 2004; PanterBrick, 2010). En lo que concierne al papel de la edad, los datos han resultado menos consistentes (Porter \& Haslam, 2005; Williams, 2007). No obstante, en su revisión de la literatura empírica Murthy (2007) concluye que los adolescentes están en mayor riesgo que los niños más pequeños para desarrollar problemas psicológicos al ser expuestos a la guerra, teniendo mayor participación fuera del hogar y mejores habilidades cognitivas para darse cuenta de ciertos eventos y sus consecuencias. 
Mientras que muchos niños/as y adolescentes sufren síntomas de desajuste psicológico debido a la guerra, muchos de ellos se recuperan de manera natural o logran seguir funcionando socialmente y académicamente bien, a pesar de la presencia de algunos síntomas (Ehntholt \& Yule, 2006; Jones \& Kafetsios, 2002; Williams, 2007). No obstante, varios estudios longitudinales han demostrado que en ciertos niños/as y adolescentes, los síntomas continúan afectando negativamente su funcionamiento y relaciones interpersonales (Barenbaum et al., 2004; Sharon, Levav, Brodsky, Shemeh \& Kohn, 2009).

\section{LAS CONSECUENCIAS DE LA GUERRA EN LA VIDA DIARIA Y SU IMPACTO PSICOLÓGICO}

Tomando en cuenta la gran diversidad individual, constatada con respecto al funcionamiento psicológico en niños/as y adolescentes afectados por la guerra, recientemente se ha empezado a mostrar más interés en la búsqueda de factores contextuales en la vida de las víctimas que favorecen u obstaculizan reacciones resilientes (Betancourt \& Kahn, 2008). Desde una perspectiva socio-ecológica (Bronfenbrenner, 1979) la violencia experimentada a nivel de la comunidad, sus consecuencias para la vida cotidiana -en el hogar, la escuela o el barrio- así como también, los recursos o factores de riesgo presentes en la comunidad, en la familia o en el individuo, interactúan y van determinando el funcionamiento y bienestar del mismo (Betancourt \& Kahn, 2008; Kienzler, 2008).

En el marco del modelo de Bronfenbrenner (1979), es necesario ir más allá del simple estudio de los efectos directos de la violencia sobre el funcionamiento psicológico del individuo, para poder comprender cómo la guerra afecta la salud mental de una población (Kienzler, 2008;Williams, 2007). La guerra afecta también las condiciones diarias en las cuales se desarrollan los seres humanos -típicamente caracterizadas por penuria económica, ruptura familiar, exclusión social, inestabilidad e inseguridad-causando aflicciones adicionales y simultáneamente socavando las estrategias y recursos disponibles para afrontar los problemas y el estrés (Betancourt \& Kahn, 2008; Miller \& Rasmussen, 2010).
El reconocimiento señalado precedentemente, dio paso al estudio de los efectos de los estresores diarios en situaciones afectadas por un conflicto armado, conceptualizados como las molestias asociadas con la penuria económica, la inseguridad y la exclusión social, por ejemplo: el estar excluido de la familia por un embarazo extramatrimonial, o el estar excluido de la sociedad por ser ex niño soldado (Miller \& Rasmussen, 2010). Previamente, este tipo de estresores ha sido asociado con la incidencia de depresión en niños/as y adultos refugiados viviendo en Europa y los Estados Unidos (Ellis, MacDonald, Lincoln \& Cabral, 2008; Heptinstall, Sethna \& Taylor, 2004; Miller et al., 2002). Aunque el estudio de estresores diarios en zonas de guerra en curso es muy reciente y se ha enfocado casi solamente en poblaciones adultas, en su revisión de la literatura Miller \& Rasmussen (2010) concluyen que hay evidencias para el valor agregado de esta variable. Encontraron que, en comparación con el modelo simple y directo de la relación "experiencias violentas $\rightarrow$ desajuste psicológico", al agregar la variable "estresores diarios" (constituyendo así un modelo con doble causalidad) mejora significativamente el valor explicativo del modelo y sistemáticamente debilita la relación directa entre violencia experimentada y salud mental.

En nuestro estudio sobre el impacto psicológico del desplazamiento forzado en adolescentes -chicas y varones- afectados por la guerra en la República Democrática del Congo, se encontró que el nivel elevado de síntomas del TEPT en adolescentes desplazados, frente a adolescentes no desplazados, se explicaba por sus elevados niveles de estresores diarios experimentados, incluso cuando eran controlados por eventos violentos (Mels, Derluyn, Broekaert \& Rosseel, 2010a). No obstante, comparando los resultados de estas dos variables -estresores diarios y la exposición a eventos violentosen datos recogidos en víctimas de guerra, se ha propuesto la hipótesis de que ambas variables afectan por la vía de trayectorias diferentes, a los síntomas del TEPT y a los síntomas internalizantes (Heptinstall, Sethna \& Taylor, 2004; Miller et al., 2002; Miller \& Rasmussen, 2010). Consecuentemente, nos interesa investigar cómo, la exposición a la violencia y los estresores diarios afectan a las conductas internalizadas, reportadas en la muestra de adolescentes afectados por la guerra en la República Democrática del 
Congo. Por lo tanto, los objetivos concretos de este estudio son los siguientes:

1) conocer la prevalencia de síntomas internalizantes y externalizantes en adolescentes viviendo en este contexto específico y

2) explorar cuáles de los factores de interés (sexo, edad, exposición violenta y estresores diarios) predicen los síntomas internalizantes y de qué manera. Con esto, esperamos contribuir al conocimiento sobre el tema y brindar información que pueda ser útil en las intervenciones, para fomentar conductas resilientes.

Basándonos en la escasa literatura sobre estresores diarios y eventos violentos en adultos, residiendo en zonas de guerra y niños/as y adolescentes refugiados asentados en el Oeste, proponemos las hipótesis siguientes:

- La exposición a la violencia y a los estresores diarios, varían según sexo y edad: los adolescentes varones estarán más expuestos que las adolescentes mujeres; y a su vez ambos -en el grupo de mayor edad- estarán más expuestos que los integrantes del grupo de menor edad.

- Los puntajes para conductas internalizadas, se asocian positivamente con las siguientes variables: mayor edad, sexo femenino, exposición a la violencia y estresores diarios.

- En los puntajes de las conductas internalizadas, los estresores diarios tienen mayor efecto que la exposición a la violencia.

\section{MÉTODO}

\subsection{Escenario de investigación}

Desde el año 1996 la República Democrática del Congo (RDC) ha sido el escenario de un conflicto complejo, involucrando a varios actores locales, nacionales e internacionales (Vlassenroot \& Raeymaekers, 2004). A pesar de un acuerdo de paz obtenido en 2003 y la consiguiente organización de elecciones democráticas, el este del país ha seguido estando muy inestable y violento, informándose de varios focos de violencia interétnica y ocupaciones extranjeras (Auteserre, 2009, Mels, Derluyn, Broekaert \& Vlassenroot, 2012). El International Rescue Committee (IRC, 2008) estimó que la guerra había causado 5.4 millones de muertos directos e indirectos, entre agosto 1998 y abril 2007. Hasta el día de hoy las Naciones Unidas están presentes en la RDC para imponer el orden, cabe destacar que hay una gran cantidad de tropas uruguayas en su misión de paz (United Nations, 2012).

En este estudio nos enfocamos en Ituri, una provincia en el noreste de la RDC, severamente afectada por la guerra étnica que tuvo su clímax entre 1999 y 2006, incentivando el despliegue de la misión de paz de las Naciones Unidas, por lo extremo de las atrocidades reportadas y sus repercusiones para el resto del país (Mels et al., 2012). Durante el conflicto en Ituri la población civil fue el blanco de masacres, mutilaciones, violaciones, torturas, asaltos e incendios, causando unos 60.000 muertos y 500.000 refugiados (Human Rights Watch, 2003).

\subsection{Instrumentos}

Aunque el estudio de poblaciones afectadas por la guerra ya tiene algunos años de historia, en la última década se develaron varios problemas metodológicos que limitan los hallazgos logrados. Entre los desafíos más profundos se encuentran los temas de validez transcultural y la relevancia contextual (Barenbaum et al., 2004; Hollifield et al., 2002; Panter-Brick, 2010). Se ha encontrado que la conceptualización de varios trastornos psicológicos -como el estrés postraumático, la depresión, la ansiedad y los trastornos de conducta- no se puede considerar como válida universalmente a través de varias culturas (Bracken, Giller \& Summerfield, 1995; Patel, Flisher, Nikapota \& Malhotra, 2008). En consecuencia, va surgiendo un cierto consenso que no se puede evaluar la salud mental en poblaciones no-occidentales, sin tomar en cuenta expresiones locales de malestar psicológico (Barenbaum et al., 2004; Kienzler, 2008; Patel et al., 2008). En consecuencia, la adaptación de instrumentos para la evaluación de síntomas psicológicos (tipo screening) para uso en otras culturas, tiene que ir más lejos que la mera traducción de los cuestionarios, incluyendo también una investigación de la validez y relevancia de los conceptos subyacentes para la población a investigar. Además, hay que verificar la viabilidad de los procedimientos de investigación usados comúnmente, sobre todo cuando se trata de poblaciones de bajos 
recursos, evitando así problemas prácticos que pueden afectar la fiabilidad de la encuesta. No obstante, el desarrollo o la adaptación transcultural y la validación de instrumentos de investigación constituyen procesos costosos que además consumen mucho tiempo, casi imposibles de llevar a cabo bajo los desafíos característicos de situaciones de guerra: peligros para la seguridad y el acceso limitado a la población -lo cual complica un muestreo representativo- y la falta de recursos (tal como la escasez de profesionales y la ausencia de instrumentos diagnósticos) (Bass, Ryder, Lammers, Mukaba \& Bolton, 2008; Patel et al., 2008).

Tomando en cuenta estas dificultades, desarrollamos una metodología para la adaptación transcultural de instrumentos tipo screening, descrito con detalle en Mels et al. (2010b). Combinamos: (1) una investigación cualitativa dirigida a un análisis de la relevancia cultural de los ítems y conceptos subyacentes a través de grupos de discusión, con informantes locales, a la vez que se proporcionaron alternativas, con (2) el estudio cuantitativo psicométrico, incluyendo un análisis factorial y de correlaciones para confirmar relaciones teóricas. Aplicamos este procedimiento -entre otros-en el instrumento siguiente que corresponde a las variables dependientes del estudio.

El Hopkins Symptoms Checklist 37 for Adolescents-HSCL-37A; Lista de Síntomas de Hopkins - versión 37 para Adolescentes- (Bean, Derluyn, Eurelings-Bontekoe, Broekaert \& Spinhoven, 2007); es una escala que mide síntomas basados en los criterios del DSM-IV: depresión (15 ítems), ansiedad (10 ítems) y perturbaciones en comportamientos externalizados (12 ítems). Este instrumento es una modificación multicultural del HSCL-25 (Derogatis, Lipman, Rikels, Ulenhoth \& Colvi, 1974), adaptada a la vida adolescente. Treinta y siete ítems evalúan la severidad de los síntomas en una escala Likert de 1 hasta 4 (nunca $=1$; a veces $=2$; seguido $=$ 3 ; siempre $=4)$. La escala está disponible en 19 idiomas, con valores adecuados de validez de constructo, contenido y criterio y una estructura bifactorial (internalización - externalización), obtenidos en una muestra de adolescentes inmigrantes y autóctonos holandeses y flamencos (Bean et al., 2007).
Para el estudio, en la versión congolesa del cuestionario, se cambiaron cuatro ítems con poca relevancia local por ítems más apropiados a la cultura, según informantes claves (Mels et al., 2010b), indicado en la tabla 1.

Tabla 1.

Puntaje promedio para los ítems del HSCL-37A, M (DS) e ítems cambiados

\begin{tabular}{rr}
\hline Conductas internalizadas & $\begin{array}{r}\text { Tota } \\
\mathbf{n}=991\end{array}$ \\
\hline
\end{tabular}

Te asustas así porque sí $1.84(.95)$

Te sientes intranquilo, no te puedes quedar quieto/a $1.82(.92)$

Te sientes angustiado/a $1.81(.96)$

Te culpas por lo que te ocurre $1.49(.75)$

$\begin{array}{ll}\text { Te mareas } & 1.73(.87)\end{array}$

Te pones a llorar con facilidad $\quad 1.67(.90)$

Te sientes nervioso/a $1.69(.86)$

Tienes poca energía $\quad 1,77(.90)$

Sufres latidos fuertes de corazón $\quad 1.79(.94)$

Tienes pocas ganas de comer $1.92(.88)$

$\begin{array}{ll}\text { Tiemblas } & 1.59(.76)\end{array}$

Tienes problemas para dormirte o te despiertas mucho

durante la noche

Te sientes tenso/a

$1.64(.76)$

Te sientes sin esperanza por el futuro $1.65(.92)$

No tienes ganas de hacer cosas $\quad 1.81(.90)$

Tienes dolor de cabeza $\quad 2.28(.91)$

$\begin{array}{ll}\text { Te sientes triste } & 2.15(.97)\end{array}$

Sufres ataques de miedo $\quad 1.83(.91)$

Te sientes solo/a $\quad 1.72(.90)$

Piensas en quitarte la vida $\quad 1.39$ (.78)

Estás sobrecargado de preocupaciones a $1.97(.99)$

Piensas demasiado en las cosas $\quad 1.87(.97)$

Tienes el sentimiento de que todo es muy difícil 1.85 (.99)

Tienes sentimientos de que no vales nada $1.59(.88)$

Hablar solo $^{\text {a }} \quad 1.50(.77)$

Conductas externalizadas

Te enojas rápidamente $\quad 1.92(.89)$

Tomas alcohol $1,15(.47)$

Amenazas a otros $\quad 1.48(.68)$

Fumas cigarrillos $\quad 1.09(.40)$

Rompes o destruyes cosas de otros $\quad 1.23(.55)$

Empiezas las peleas $\quad 1.27(.63)$

Haces mal a otro a propósito $\quad 1,29(.60)$

Te peleas $1.46(.71)$

No tener interés en los estudios a ${ }^{a} \quad 1.65$ (1.04)

No respetar las reglas (de tus padres, profesores) ${ }^{\mathrm{a}} \quad 1.50(.81)$

Robas cosas $1.16(.50)$

Usas drogas (marihuana, cocaína, pasta base, otros) 1.11 (.47)

a ítems cambiados por razones culturales

El siguiente estudio de validación -llevado a cabo en una muestra de 1046 adolescentes congoleses de ambos sexos, de entre 13 y 21 años- confirmó los valores de fiabilidad interna (Cronbach's Alphas de .90 para la escala en francés y .91 para la versión en swahili) y la estructura factorial previamente encontrada por Bean et al. (2007). 
No obstante, la evaluación de los valores de fiabilidad interna de las sub-escalas mostró una fiabilidad buena para la sub-escala de internalización (.89 para la escala francesa y .91 para la escala swahili), pero discutible para la sub-escala de externalización (.76 para la escala francesa y .60 para la escala swahili). Por esta razón, en este artículo, nos enfocaremos, solamente en el análisis de covarianza de la sub-escala de conductas internalizadas.

Para investigar las variables independientes, desarrollamos -igualmente basándonos en el estudio cualitativo descrito en Mels et al. (2010b) - una escala socio-demográfica y los dos cuestionarios siguientes:

El Adolescent Complex Emergency Exposure Scale (Escala Adolescente de Exposición en Emergencias Complejas) (Mels et al., 2009). Se trata de una lista de 13 eventos impactantes y potencialmente traumáticos, vinculados específicamente a la guerra (como la separación de la familia, la muerte de familiares o amigos, ser testigo de asesinato, experimentar ataques armados, etc.). Para cada uno de los eventos en la lista, el participante debe indicar si lo experimentó o no (respuesta cerrada: si/no). La suma total de todos los ítems lleva a un valor para eventos impactantes acumulados, o sea: exposición a la violencia.

El Adolescent Complex Emergency Daily Stressors Scale (Escala Adolescente de Estresores Diarios en Emergencias Complejas) (Mels et al. 2010a), evalúa 13 dificultades sufridas en el último mes (por ejemplo: insuficiencia de comida, cuidados médicos, etc.) y cinco condiciones estresantes conocidas por impactar negativamente en la vida diaria (por ejemplo, rechazo de la familia, matrimonio forzado, etc.).
Se indica la respuesta cerrada marcando si o no. La suma de todas las dificultades reportadas opera como indicador para los estresores diarios sufridos.

\subsection{Participantes}

Entre noviembre 2007 y febrero 2008 se realizó una encuesta en 13 colegios secundarios a través de cuatro zonas seleccionadas de la provincia (Bunia, Rwampara, Nyankunde y Rethy). Se seleccionaron las zonas según los criterios siguientes: accesibilidad física y ofrecer un mínimo de seguridad para llevar a cabo la encuesta, representatividad de aéreas rurales, periféricas y urbanas (o sea la variable zona de residencia). Participaron 1051 alumnos entre 13 y 21 años, asistiendo entre el primer y cuarto año (a causa de la guerra se suspendieron las clases, por lo que el sistema escolar tiene una dispersión muy amplia de edades por años escolares). Ninguno de los alumnos se negó a la participación. No obstante, los datos recogidos de 60 participantes resultaron deficientes para el testeo de las hipótesis, reduciendo el tamaño de la muestra a 991. Las características de la muestra y su distribución según sexo, grupo de edad y zona de residencia se presentan en la Tabla 2.

\subsection{Procedimiento}

La encuesta consistió en la administración en clase de los cuestionarios auto-informados referidos, junto con la explicación del estudio y la obtención de su consentimiento informado en forma escrita. Por las altas tasas de analfabetismo esperadas entre los padres, resultó imposible conseguir su consentimiento escrito para la participación de sus hijos en la investigación.

Tabla 2.

Características de los participantes, No. (\%)

\begin{tabular}{lccc}
\hline & $\begin{array}{c}\text { Chicas } \\
\boldsymbol{n}=\mathbf{4 4 9}\end{array}$ & $\begin{array}{c}\text { Varones } \\
\boldsymbol{n = 5 4 2}\end{array}$ & $\begin{array}{c}\text { Total } \\
\boldsymbol{n}=\mathbf{9 9 1}\end{array}$ \\
\hline Edad en años & & & \\
$13-14$ & $132(29.4)$ & $115(21.2)$ & $247(24.9)$ \\
$15-16$ & $198(44.1)$ & $236(43.5)$ & $434(43.8)$ \\
$17-21$ & $119(26.5)$ & $191(35.2)$ & $310(31.3)$ \\
$\begin{array}{l}\text { Zona de residencia } \\
\text { Rural }\end{array}$ & $114(25.4)$ & $134(24.7)$ & $248(25.0)$ \\
$\quad$ Urbano & $178(39.6)$ & $246(45.4)$ & $424(42.8)$ \\
$\quad$ Periferico & $157(35.0)$ & $162(29.9)$ & $319(32.2)$ \\
Total & $449(45.3)$ & $542(54.7)$ & \\
\hline
\end{tabular}


Se ofreció la elección entre una batería en Francés o Swahili para dejar a los alumnos la posibilidad de elegir el idioma con el cual se sintieran más cómodos. Se les proporcionó información y datos de contacto de servicios psicosociales a los participantes, para poder cumplir con necesidades psicológicas que pudieran surgir en algunos alumnos durante su participación. Los investigadores (la autora extranjera y un asistente congolés) estuvieron presentes para responder las preguntas o dudas que surgieran durante la administración.

Como en aquel momento no había una instancia activa en la RDC que autorizace la investigación, se buscó y obtuvo la aprobación del Comité de Ética de la Facultad de Psicología y Ciencias Pedagógicas de la Universidad de Gante en Bélgica, donde la autora estaba como becaria doctoral en el momento de la investigación.

\section{ANÁLISIS ESTADÍSTICOS}

Se utilizaron estadísticos descriptivos para reseñar las características de la muestra según sexo, promedios de la exposición violenta, estresores diarios y conductas internalizadas y externalizadas -según sexo grupo de edad y zona de residencia-. Las diferencias para los promedios por grupos de edad y sexo, fueron comprobados con one-way ANOVA (con Games-Howell, post-hoc tests) y t-test respectivamente. Se usó ANCOVA para investigar como las variables sexo, edad, estresores diarios y exposición violenta, mediaban en los puntajes de conductas interiorizadas. El modelo testeado contuvo todos los efectos directos y efectos de interacción con las variables estresores diarios y exposición violenta, con el objetivo de investigar sus efectos potencialmente acumulativos. También estaban incluidos los efectos directos e indirectos de la variable zona de residencia.

En un estudio previo (Mels et al., 2009), ésta variable se mostró como moderador relevante en la prevalencia de los síntomas del TEPT. Los test de Levene confirmaron la equivalencia de varianzas entre los grupos evaluados. Los datos fueron analizados con SPSS 15.0 Statistical Software (SPSS Inc., 2006).
El nivel de significación para las comparaciones entre promedios por grupos fue de a $p \leq .001$. Para la interpretación del modelo ANCOVA aplicamos $p<.05$ como umbral para estudio de significación. Se usó la eta parcial al cuadrado $\left(\eta^{2}\right)$ como una aproximación del efecto del tamaño (efect size).

\section{RESULTADOS}

\subsection{Conductas internalizadas y externalizadas}

La tabla 1 presenta los ítems del HSCL-37A según las sub-escalas internalización - externalización y los puntajes promedios en la muestra (promedio en una escala Likert de 1 a 4). Con respecto a las conductas internalizadas reportadas, Ilaman la atención los altos puntajes para los ítems "tener dolor de cabeza" y "sentirse triste". Desde el tercer hasta quinto lugar de síntomas más frecuentemente reportados encontramos los ítems "estar sobrecargado de preocupaciones", "tener problemas para dormirse o despertarse durante la noche", y "tener pocas ganas de comer". Los cinco síntomas con mayores puntajes en promedio se encuentran entre los criterios para el diagnóstico de depresión.

En comparación, los ítems de la sub-escala externalización tienen promedios menores. Un solo ítem llama la atención por su promedio alto: "enojarse rápidamente".

La tabla 3 (página siguiente) muestra los promedios de las sub-escalas del HSCL-37A, y compara los promedios según grupo de edad, sexo y zona de residencia. No encontramos diferencias entre los grupos en conductas externalizadas. En lo que concierne a las conductas interiorizadas, se encontraron diferencias significativas entre los grupos: las niñas reportaron más conductas internalizadas que los varones, y los adolescentes mayores, en general reportaron más síntomas que los adolescentes más jóvenes.

\subsection{Exposición a la violencia y estresores diarios}

Las medias de las variables exposición a la violencia y estresores diarios, según sexo y 
Tabla 3.

Conductas internalizadas y externalizadas en la muestra

\begin{tabular}{lcccccc}
\hline & \multicolumn{2}{c}{ Conductas internalizadas } & \multicolumn{3}{c}{ Conductas externalizadas } \\
\hline & M (DS) & Estadística & $p$ & M (DS) & Estadística & $p$ \\
\hline $\begin{array}{l}\text { Total } \\
\text { Sexo }\end{array}$ & $1.74(.49)$ & & & $1.34(.31)$ & & \\
Chicas & & $\mathrm{t}(989)=3.40$ & .001 & & $\mathrm{t}(989)=-.32$ & .752 \\
Varones & $1.80(.52)$ & & & $1.33(.31)$ & & \\
Edad en años & $1.69(.46)$ & & & & \\
$13-14$ & & $\mathrm{~F}(2,988)=11.58$ & .000 & & $\mathrm{~F}(2,988)=2.18$ & .114 \\
$15-16$ & $1.62(.43)$ & & & $1.31(.27)$ & & \\
$17-21$ & $1.75(.51)$ & & & $1.36(.32)$ & & \\
Zona de residencia & $1.82(.51)$ & & & & \\
Rural & & $\mathrm{F}(2,988)=11.06$ & .000 & & & \\
Urbana & $1.69(.43)$ & & & $1.30(.26)$ & & \\
Periférica & $1.82(.55)$ & & & $1.33(.32)$ & & \\
\hline
\end{tabular}

grupo de edad, están presentadas en la tabla 4. Los datos muestran que no había diferencias en los estresores diarios experimentados según sexo. Sí variaron significativamente según edad: los adolescentes mayores -de ambos sexos- reportaron más estresores diarios que sus pares de menor edad.

Con respecto a la exposición a la violencia, encontramos diferencias significativas según edad y según sexo: los adolescentes varones resultaron más expuestos que las chicas, también los adolescentes (varones y mujeres) de mayor edad reportaron más eventos violentos experimentados, que los de menor edad -de ambos sexos-.

\subsection{Predictores de conductas internalizadas}

El modelo comprobado explicó el $41 \%$ de la varianza en la variable independiente $\left(R^{2}=.411\right)$. Se encontraron pequeños efectos en relación con la zona de residencia $(F(2,990)$ $\left.=3.643, p=.027, \eta^{2}=.007\right)$ y estresores diarios $\left(F(2,972)=6.425, p=.011, \eta^{2}=.007\right)$, que no obstante deben ser matizados a la luz de los efectos de interacción comprendidos en estas variables.

El efecto con mayor valor $\eta^{2}$ en el modelo, fue el efecto de interacción entre exposición violenta y estresores diarios $(F(1,972)=6.425$, $\left.p=.000, \eta^{2}=.027\right)$ indicando comportamientos internalizados elevados a medida que ambas variables acumulaban $(B=.006, S E=.001$, $t=4.916, p=.000)$.

También se constataron efectos de interacción pequeños entre sexo y estresores diarios $\left(F(1,972)=7.808, p=.005, \eta^{2}=.008\right)$ y entre zona de residencia y exposición a la violencia $\left(F(1,972)=5.421, p=.005, \eta^{2}=.011\right)$ : las chicas tienen más conductas internalizadas que los adolescentes varones bajo la acumulación de estresores diarios $(B=.021$, SE $=.008$, $t=2.794, p=.005)$. En aéreas periféricas la acumulación de eventos violentos afecta más negativamente a las conductas internalizadas que en otras aéreas $(B=.036, S E=.014$, $t=2.577, p=.010$ ).

Tabla 4.

Exposición a la violencia y estresores diarios en la muestra

\begin{tabular}{|c|c|c|c|c|c|c|}
\hline & \multicolumn{3}{|c|}{ Exposición a la violencia } & \multicolumn{2}{|c|}{ Estresores diarios } & \multirow[b]{2}{*}{$p$} \\
\hline & M (DS) & Estadística & $p$ & M (DS) & Estadística & \\
\hline Total & $4.72(2.70)$ & & & $6.95(3.78)$ & & \\
\hline Sexo & & $\mathrm{t}(989)=-3.95$ & .000 & & $\mathrm{t}(989)=-1.28$ & .201 \\
\hline Chicas & $4.35(2.57)$ & & & $6.76(3.81)$ & & \\
\hline Varones & $5.03(2.77)$ & & & $7.07(3.76)$ & & \\
\hline Edad en años & & $\mathrm{F}(2,988)=48.72$ & .000 & & $F(2,988)=21.39$ & .000 \\
\hline $13-14$ & $3.72(2.30)$ & & & $5.87(3.59)$ & & \\
\hline $15-16$ & $4.49(2.70)$ & & & $6.82(3.95)$ & & \\
\hline $17-21$ & $5.83(2.62)$ & & & $7.92(3.44)$ & & \\
\hline Zona de residencia & & $\mathrm{F}(2,988)=16.31$ & .000 & & $F(2,988)=21.64$ & .000 \\
\hline Rural & $5.20(2.40)$ & & & $7.26(3.48)$ & & \\
\hline Urbana & $5.10(2.85)$ & & & $7.57(3.82)$ & & \\
\hline Periférica & $3.81(2.51)$ & & & $5.82(3.73)$ & & \\
\hline
\end{tabular}




\section{DISCUSIÓN Y CONCLUSIONES}

Mientras que algunos/as adolescentes desarrollan reacciones psicopatológicas duraderas, el desajuste psicológico en jóvenes afectados por la guerra está cada vez más reconocido como una reacción aguda, pero temporal, frente a eventos extremadamente estresantes. Generalmente esta reacción inicial está seguida por una fase de recuperación en la cual, niños/as y adolescentes resilientes vuelven a patrones de emociones y comportamientos normales, con el paso del tiempo (Williams, 2007). Tomando en cuenta estas diferencias observadas, es importante desarrollar un mejor entendimiento sobre el impacto de varios factores de riesgo y factores protectores (tales como la edad, sexo, la cantidad de experiencias violentas sufridas y estresores diarios) y cómo interactúan entre ellos. Estos conocimientos nos ayudarán a fomentar la estimulación de la resiliencia y la prevención de problemas psicológicos duraderos en niños/as y adolescentes en varias situaciones amenazadas por la violencia (Betancourt \& Kahn, 2008; Williams, 2007).

Este estudio, en particular se enfocó en la exploración de dos factores de riesgo asociados con la vida en zonas de guerra -la exposición a eventos violentos y los estresores diarios (las consecuencias cotidianas de la pobreza y la exclusión social)- y sus relaciones directas e indirectas con conductas internalizadas.

Confirmando estudios anteriores, en esta investigación, los adolescentes varones reportaron haber experimentado un mayor número de eventos violentos que las adolescentes mujeres; y a su vez ambos reportaron una mayor exposición en comparación con los/las adolescentes de menor edad (Barenbaum et al., 2004; Macksoud \& Aber, 1996; Mels et al., 2009; Murthy, 2007). Se ha atribuido este resultado a la mayor independencia en la sociedad y el menor grado de protección por parte de los padres hacia los hijos/as adolescentes mayores por un lado y a los hijos varones frente a las hijas mujeres de menor edad, por otro (Murthy, 2007; Porter \& Haslam, 2005). Si bien, respecto a los estresores diarios, también se podría atribuir que la menor protección por parte de los padres hacia los adolescentes varones, llevará a encontrar diferentes niveles de estresores diarios sufridos por éstos en comparación con las chicas, los datos no confirmaron esta hipótesis ya que ambos presentaron niveles de estresores diarios semejantes. Se podría suponer entonces, que en un país tan indigente y tan aquejado por las tensiones étnicas como la República Democrática del Congo, la pobreza y la exclusión social son tan generalizadas que afectaran a todos, independientemente de su posición participativa en la sociedad.

Aunque este estudio no tenía el objetivo de comparar los puntajes obtenidos en la muestra congolesa con otras poblaciones no afectadas por la guerra, podemos concluir que las medias obtenidas en la sub-escala de comportamientos internalizados del HSCL-37A son altas. En estudios previos en Europa y Estados Unidos, se ha establecido el valor 1.75 como umbral indicativo para la significancia clínica de la sintomatología (Derogatis et al., 1974; Hollifield et al., 2002). Aunque las poblaciones refiridas, ni los umbrales extraídos de ellas son comparables con la población investigada, en este estudio llama la atención que el promedio total de nuestra muestra (1.74) casi llega a este puntaje. No obstante es dudoso que, en esta muestra, los síntomas tengan implicaciones clínicas comparables con aquellas encontradas en zonas desarrolladas y pacíficas (Bracken et al., 1995; Jones \& Kafetsios, 2002; Miller\& Rasmussen, 2010).

De acuerdo con nuestra hipótesis, los puntajes para problemas de conductas internalizadas se asocian positivamente con el sexo femenino, la exposición a la violencia y estresores diarios. No obstante estos efectos constatados exigen cierta explicación: las adolescentes mujeres son más vulnerables que los adolescentes varones, tienen mayor presencia de síntomas internalizantes, pero solamente aquellas con mayores índices de estresores diarios sufridos. Este dato puede indicar que la resiliencia de las chicas, se ve negativamente afectada debido a los mayores niveles de estrés diario, conclusión ésta, similar a la alcanzada por Miller et al. (2008) en su estudio con mujeres afganas.

En lo que concierne a los efectos respectivos de la exposición a la violencia y estresores diarios en los puntajes de conductas internalizadas, podemos concluir que -como se ha planteado- se encontró mayor influencia de estresores diarios, aunque este efecto no puede estar completamente separado del impacto de 
los eventos violentos sufridos. Aunque haya un efecto directo negativo, pequeño, de estresores diarios en el reporte de síntomas internalizantes, la mayor parte de la varianza fue explicada por el efecto indirecto de estresores diarios, en interacción con la exposición a eventos violentos. Esto implica que, la acumulación de estresores diarios tiene mayor efecto negativo para la salud mental, cuando está acompañado por mayores niveles de exposición a eventos violentos. En otras palabras: mientras que mayores niveles de estresores diarios están asociados con mayores niveles de conductas internalizadas, sobre todo en adolescentes mujeres, la exposición a la violencia es facilitador en este proceso, aumentando el efecto negativo de los estresores diarios en el ajuste psicológico.

Este resultado corresponde con las conclusiones de Miller et al. (2002) en su estudio de refugiados bosnios adultos: mientras que la exposición a eventos violentos resultó altamente predictiva para la aparición de síntomas de TEPT, la sintomatología depresiva se vio principalmente afectada por estresores diarios. No obstante, en los casos más graves, la exposición a eventos violentos también explican los síntomas de depresión. Este proceso se ve reflejado en las altas tasas de depresión -entre otros síntomas internalizantes- constatadas a través de varias poblaciones afectadas por la guerra (Barenbaum et al., 2004; Ehntholt \& Yule, 2006; Lynch, 2003; Murthy, 2007). Vale plantear la cuestión de, en qué medida, se puede seguir concibiendo la violencia crónica como un evento extraordinario (uno de los criterios usados en el diagnostico de TEPT) en situaciones que ha tomado más un carácter de una molestia diaria, y lo que esta implica para la sintomatología (Kienzler, 2008; Miller \& Rasmussen, 2010).

Mientras que en este trabajo se encontraron diferencias significativas en conductas internalizadas según edad, el análisis de covarianza no dio evidencia de una mayor vulnerabilidad para el desarrollo de síntomas en ciertas edades. Frecuentemente se han constatado diferencias en niveles de ajuste psicológicos según edad, no obstante con conclusiones muy inconsistentes (Barenbaum et al., 2004; Porter \& Haslam, 2005). Más que una mayor vulnerabilidad directa para el desarrollo de síntomas en adolescentes mayores, parece ser más probable que se trate de una mayor vulnerabilidad por verse expuestos tanto a estresores diarios, como a eventos violentos. La consecuencia lógica es entonces el mayor desajuste psicológico en estos adolescentes, lo cual no significa que sean menos resilientes bajo niveles de estrés comparables (Williams, 2007).

\subsection{Limitaciones del estudio e implicaciones futuras}

Primero, se destacan las condiciones extraordinarias en las cuales se llevó a cabo este estudio, limitando el rango de opciones presentes para su diseño. Cuestiones de infraestructuras empobrecidas (para la comunicación y el transporte), inseguridad y riesgos para la salud (restringiendo el acceso a ciertas areas con presencia de rebeldes y casos confirmados de cólera) complicaron la obtención de una muestra aleatoria. Asimismo, las condiciones nos dirigieron hacia un diseño transversal con el uso de solamente una fuente -los/las adolescentes- para evaluar sus conductas. En segundo lugar, para garantizar un cierto nivel de alfabetismo necesario para el buen entendimiento de los cuestionarios, optamos por una encuesta en liceos, lo cual limita la generalizabilidad de la muestra a todos los jóvenes en la región. Por último, a pesar de la importancia sobre la adaptación y validación transcultural de los instrumentos de investigación usados (Mels et al., 2010b); no se pueden excluir completamente los "errores" transculturales en este tipo de investigación (Bracken et al., 1995).

No obstante estas limitaciones, creemos que este estudio contribuye al estado del arte sobre este tema, proporcionando datos recogidos en una población muy poco investigada: los niños/ as y adolescentes viviendo en una zona de guerra (Barenbaum et al., 2004; Porter \& Haslam, 2005). Además, las poblaciones africanas están sistemáticamente menos representadas en cualquier campo de estudio en psicología (Patel et al., 2008; Porter \& Haslam, 2005). En un momento de gran desconfianza hacia los modelos tradicionales, generalmente usados en investigaciones sobre la relación entre el estar expuesto a actos violentos y reacciones psicopatológicas, este estudio colabora en el entendimiento sobre factores de riesgo y sus interacciones mutuas, y cómo afectan en síntomas internalizantes y en las reacciones resilientes. 
La confirmación del papel fundamental que juegan los estresores diarios en el ajuste psicológico de niños/as y adolescentes, que se desarrollan en situaciones afectadas por conflictos armados, tiene algunas implicaciones importantes para la intervención psicológica en dichas condiciones. Mientras que los eventos violentos sufridos durante la guerra son irreversibles en la fase de post-conflicto, la idea de que una reducción de estresores diarios puede promover la resiliencia y minimizar -en cierta medida- los síntomas psicológicos causados por el estrés acumulado durante la guerra, ofrece un punto de partida efectivo para procesos de recuperación (Miller \& Rasmussen, 2010). Esto implica que la mejora de las circunstancias socio-económicas de los hogares y comunidades en las cuales estos jóvenes se encuentran, beneficiará su desarrollo sano y adaptación psicologica.

Junto con el debate sobre la utilidad y validez de investigaciones enfocadas en el mero estudio de los efectos directos de la violencia sufrida y los síntomas psicopatológicos (principalmente el TEPT), en los últimos años se ha desplegado simultáneamente un cuestionamiento sobre la utilidad del modelo terapéutico clásico en poblaciones afectadas por conflictos armados (Mels et al., 2012). Este abordaje ha demostrado ser inefectivo por cuestiones prácticas (tal como, la falta de recursos y profesionales en estas zonas generalmente poco desarrolladas) y su validez transcultural muy limitada (Bracken et al., 1996; Patel et al., 2006), impulsando un modelo de intervención psicosocial como alternativa (Miller \& Rasmussen, 2010; PanterBrick, 2010; Williams, 2007). Este modelo propone la importancia de un abordaje amplio de problemas psicológicos, tomando en cuenta los aspectos concretos y sociales de la situación diaria que da forma al desarrollo humano. Nuestro estudio confirma el potencial de este tipo de intervenciones en zonas de guerra y da paso a futuras investigaciones, evaluando su aplicabilidad en otros contextos afectados por la pobreza y la violencia comunitaria.

\section{REFERENCIAS}

Amone-P'Olak, K., Garnefski, N., \& Kraaij, V. (2007). Adolescents caught between fires: cognitive emotion regulation in response to war experiences in Northern Uganda. Journal of Adolescence, 30 (4), 655-669.
Autesserre, S. (2009). Hobbes and the Congo: frames, local violence, and international intervention. International Organization, 63 (2), 249-280.

Barenbaum, J., Ruchkin, V., \& Schwab-Stone, M. (2004). The psychosocial aspects of children exposed to war: practice and policy initiatives. Journal of Child Psychology \& Psychiatry, 45 (1), 41-62.

Bass, J. K., Ryder, R. W., Lammers, M. C., Mukaba, T. N., \& Bolton, P. A. (2008). Postpartum depression in Kinshasa, Democratic Republic of Congo: Validation of a concept using a mixed- methods cross-cultural approach. Tropical Medicine and International Health, 13, 1534-1542.

Bean, T., Derluyn, I., Eurelings-Bontekoe, E., Broekaert, E., \& Spinhoven, P. (2007). Validation of the multiple language versions of the Hopkins Symptom Checklist-37 for Refugee Adolescents. Adolescence, 42, 51-71.

Betancourt, T., \& Khan, K. (2008). The mental health of children affected by armed conflict: protective processes and pathways to resilience. International Review of Psychiatry, 20 (3), 317-328.

Bracken, P., Giller, J., \& Summerfield, D. (1995). Psychological responses to war and atrocity: the limitations of current concepts. Social Science \& Medicine, 40, 1073-1082.

Bronfenbrenner, U. (1979). The ecology of human development. Cambridge: Harvard University Press.

Derluyn, I., Broekaert, E., Schuyten, G., \& De Temmerman, E. (2004). Post-traumatic stress in former Ugandan child soldiers. Lancet, 363 (5), 861-863.

Derluyn, I., Mels, C., \& Broekaert, E. (2009). Mental health problems in separated refugee adolescents. Journal of Adolescent Health, 44 (3), 292-297.

Derogatis, L., Lipman, R., Rikels, K., Ulenhoth, E., \& Colvi, L. (1974). The Hopkins Symptom Checklist (HSCL): a selfreportsymptom inventory. Behavioural Science, 19, 1-15.

Ehntholt K., \& Yule, W. (2006). Practitioner review: assessment and treatment of refugee children and adolescents who have experienced war-related trauma. Journal of Child Psychology \& Psychiatry, 47 (12), 1197-1210.

Ellis, H., MacDonald, H., Lincoln, A., \& Cabral, H. (2008). Mental health of Somali adolescent refugees: the role of trauma, stress and perceived discrimination. Journal of Consulting \& Clinical Psychology, 76 (2), 184-193.

Heptinstall, E., Sethna, V., \& Taylor, E. (2004). PTSD and depression in refugee children - Associations with pre-migration trauma and post-migration stress. European Child \& Adolescent Psychiatry, 13 (6), 373-380.

Hollifield, M., Warner, T., Lian, N., Krakow, B., Jenkins, J., Kesler, J.,...Stevenson,J.(2002). Measuring trauma and health status in refugees. A critical review. Journal of the American Medical Association, 288, 611-621.

Human Rights Watch (2003). Covered in blood. Ethnically targeted violence in Northern DRC. New York: HRW Publications. Recuperado de http://www.hrw.org/en/ reports/ 2003/07/07/covered-blood-0

International Rescue Committee (2008). Mortality in the Democratic Republic in Congo: an ongoing crisis. New York: International Rescue Committee. 
Jones, L., \& Kafetsios, K. (2002). Assessing adolescent mental health in war-affected societies: the significance of symptoms. Child Abuse \& Neglect, 26 (10), 1059-1080.

Kienzler, H. (2008). Debating war-trauma and post-traumatic stress disorder (PTSD) in an interdisciplinary arena. Social Science \& Medicine, 67, 218-227.

López-Soler, C., Castro, M., Alcántara, M., Fernández, V., Prieto, M., Puerto, J. C., Daset, L. R. (2008). Consecuencias del maltrato grave intrafamiliar en la infancia: conceptualización u diagnostico de las reacciones postraumáticas complejas. Ciencias Psicológicas, 2 (2), 103-118.

Lynch, M. (2003). Consequences of children's exposure to community violence. Clinical Child and Family Psychology Review, 6 (4), 265-274.

Macksoud, M., \& Aber, J. (1996). The war experiences and psychosocial development of children in Lebanon. Child Development, 67 (1), 70-88.

Mels, C., Derluyn, I., Broekaert, E., \& Rosseel, Y. (2009). Screening for traumatic exposure and post-traumatic stress symptoms adolescents in war-affected Eastern Democratic Republic of Congo. Archives of Pediatrics \& Adolescent Medicine, 163, 525-530.

Mels, C., Derluyn, I., Broekaert, E., \& Rosseel, Y. (2010a). The psychological impact of forced displacement and related risk factors on Eastern Congolese adolescents affected by war. Child Psychology \& Psychiatry, 51 (10), 1096-1104.

Mels, C., Derluyn, I., Broekaert, E., \& Rosseel, Y. (2010b). Community-based cross-cultural adaptation of mental health measures in emergency settings: validating the IES-R and HSCL-37A in Eastern Democratic Republic of Congo. Social Psychiatry \& Psychiatric Epidemiology, 45 (9), 899-910.

Mels, C., Derluyn, I., Broekaert, E., \& Vlassenroot, K. (2012). Exploring the context for adolescent mental heath and spychosocial assistance in the Eastern Democratic Republic of Congo. In I. Derluyn, C. Mels, S. Parmentier, W. Vandenhole, Re-Member. Rehabilitation, Reintegration and Reconciliation of War-affected Children. Volume 11 of the Series on Transitional Justice. Cambridge/Antwerp/Portland: Intersentia.

Miller, K., Weine, S., Ramic, A., Brkic, N., Djuric Bjedic, Z., Smajkic, A.,... Worthington, G. (2002). The relative contribution of war experiences and exile-related stressors to levels of psychological distress among Bosnian refugees. Journal of Traumatic Stress, 15 (5), 377-387.

Miller, K., Omidian, P., Rasmussen, A., Yaqubi, A., \& Daudzai, H. (2008). Daily stressors, war experiences, and mental health in Afghanistan. Transcultural Psychiatry, 45 (4), 611-638.

Miller, K., \& Rasmussen, A. (2010). War exposure, daily stressors; and mental health in conflict and postconflict settings: bridging the divide between traumafocussed and psychosocial frameworks. Social Science \& Medicine, 70, 7-16.

Murthy, R. (2007). Mass violence and mental health - Recent epidemiological findings. International Review of Psychiatry, 19 (3), 183-192.

Panter-Brick, C. (2010). Conflict, violence, and health: setting a new interdisciplinary agenda. Social Science \& Medicine, 70, 1-6.

Patel, V., Flisher, A., Nikapota, A., \& Malhotra, S. (2008). Promoting child and adolescents mental health in low and middle income countries. Journal of Child Psychology \& Psychiatry, 49, 313-334.

Porter, M., \& Haslam, N. (2005). Predisplacement and postdisplacement factors associated with mental health of refugees and internally displaced persons. Journal of the American Medical Association, 249, 602-612.

Project Ploughshares (2012). Armed Conflicts Report 2011. Project Ploughshares: Waterloo Ontario Canada. Recuperado de http://www.ploughshares. ca/content/2011-armed-conflicts-report

Sharon, A., Levav, I., Brodsky, J., Shemeh, A.A., \& Kohn, R. (2009). Psychiatric disorders and other health dimensions among Holocaust survivors 6 decades later. The British Journal of Psychiatry, 195, 331-335.

United Nations (2012). MONUSCO facts and figures. Recuperado de http://www.un.org/en/peacekeeping/ missions/monusco/facts.shtml

Vlassenroot, K., \& Raeymaekers, T. (2004). The politics of rebellion and intervention in Ituri: The emergence of a new political complex? African Affairs, 103 (412), 385-412.

Williams, R. (2007). The psychosocial consequences for children of mass violence, terrorism and disasters. International Review of Psychiatry, 19 (3), 263-277.

\footnotetext{
Para citar este artículo:

Mels, C. (2012). Entre pobreza y violencia: ¿cómo afectan los estresores diarios y la violencia a adolescentes en zonas de guerra? Ciencias Psicológicas VI (2): 111-122.
} 